VOL. 54 (1996) [221-240]

\title{
EMBEDDING INTO GROUPS WITH WELL-DESCRIBED LATTICES OF SUBGROUPS
}

\author{
ViatcheslaV N. ObRaztsov
}

\begin{abstract}
A thrifty embedding scheme of an arbitrary set of groups in a simple infinite group with a given outer automorphism group is presented. One of the applications of this scheme is the existence (assuming $\mathrm{CH}$ ) of an uncountable group $G$ in which all proper subgroups are countable such that $G$ contains every countable group.
\end{abstract}

\section{INTRODUCTION}

By a famous embedding theorem of Higman, Neumann and Neumann [1], every countable group can be embedded in a 2-generator group. But this embedding construction contains a lot of subgroups other than the embedding group and its conjugates, and there is little information about the automorphism group of the resulting group. On the other hand, the method of graded diagrams developed by Ol'shanskii has given an approach to constructing of difficult examples of groups such as, for example, nonabelian infinite groups all of whose proper subgroups are finite (see [8]). This technique was extended in [3] to diagrams over free products and applied to quotient groups of free products. As a result, a theorem was proved in [3] on embeddability of every countable set $\left\{G_{\mu}\right\}_{\mu \in I}$ of countable groups without involutions in a simple 2-generator infinite group $G$ in which every proper subgroup is either a cyclic group or contained in a subgroup conjugate to one of the embedding groups $G_{\mu}$, and the generalizations of this theorem to the case of arbitrary sets $\left\{G_{\mu}\right\}_{\mu \in I}$ of groups without involutions were given in $[4,5,6]$. These constructions have given an opportunity to obtain minimal extensions of the subgroup lattices of the resulting groups $G$ in comparison with the subgroup lattices of the embedding groups which was used in $[3,4,5,6]$ and $[8]$ for solution of some famous problems, in particular, a well-known problem about the existence of uncountable Artinian groups.

On the other hand, it is easy to see that these results can not be extended to the case of groups $\left\{G_{\mu}\right\}_{\mu \in I}$ with involutions, since any involution $h \in G_{\mu}$ together with any conjugate involution $g h g^{-1}, g \in G \backslash G_{\mu}$, must generate in $G$ a dihedral subgroup. Ol'shanskii [7] proved that by making such exemptions, one might avoid mentioning the

Received 26th October 1995

Supported by a grant from the Australian Research Council.

Copyright Clearance Centre, Inc. Serial-fee code: 0004-9729/96 \$A2.00+0.00. 
absence of involutions from $G_{\mu}$ in the statement of the theorem in [3]. (It also leads to the loss of the property that the resulting group $G$ may chosen to be torsion (of finite exponent) if all embedding groups are torsion (of finite exponent).) By combining the ideas from [6] and [7], we obtain the following embedding scheme of an arbitrary set of groups into a simple infinite group with a "well-described" lattice of subgroups and a given outer automorphism group.

Let $\left\{G_{i}\right\}_{i \in I}$ be an arbitrary set of nontrivial groups. We denote by $\Omega^{1}$ the free amalgam of the groups $G_{i}, i \in I$, that is, the set $\bigcup_{i \in I} G_{i}$ with $G_{i} \cap G_{j}=1$ whenever $i \neq j$. We say that the mapping $g: \Omega^{1} \rightarrow G$ is an embedding of $\Omega^{1}$ into $G$ if $g$ is injective and its restriction to every $G_{i}$ is a homomorphism.

Let $\Omega=\Omega^{1} \backslash\{1\}=\left\{a_{j}, j \in J\right\}$. Then a mapping $f: 2^{\Omega} \backslash\{\emptyset\} \rightarrow 2^{\Omega}$ is called generating on the set $\Omega$ if the following conditions hold:

1) if $C \subseteq G_{i}$ for some $i \in I$, then $f(C)=g p\{C\} \backslash\{1\}$;

2) if $C \nsubseteq G_{i}$ for each $i \in I$ and $C=\{a, b\} \subseteq \Omega$, where $a$ and $b$ are involutions (such a subset $C$ will be called dihedral), then $f(C)=C$;

3) if $C$ is a finite non-dihedral subset of $\Omega$ and $C \nsubseteq G_{i}$ for each $i \in I$, then $f(C)=B$, where $B$ is an arbitrary countable subset of $\Omega$ such that $C \subseteq B$ and if $D$ is a finite subset of $B$, then $f(D) \subseteq B$;

4) if $C$ is an infinite subset of $\Omega$, then $f(C)=\bigcup_{A \in T} f(A)$, where $T$ is the set of all finite subsets of $C$.

For example, a generating mapping $f$ on $\Omega$ can be defined in the following way: if $C \in 2^{\Omega} \backslash\{\emptyset\}$ and $C=\bigcup_{i \in I} C_{i}$, where $C_{i}=C \cap G_{i}, i \in I$, then $f(C)=\left(\bigcup_{i \in I} g p\left\{C_{i}\right\}\right) /\{1\}$.

We denote by $G(1)$ the free product of groups $G_{i}, i \in I$. A group $G$ having a presentation

$$
G=\langle G(1) \| R=1 ; R \in D\rangle
$$

is called (diagrammatically) aspherical ((diagrammatically) atoroidal) if every diagram on the sphere (torus) over (1.1) is either non-reduced or consists entirely of 0 -cells. (All necessary information about diagrams can be found in [8].)

Let $G=g p\{\Omega\}, f$ an arbitrary generating mapping on $\Omega$. We say that $X$ is a minimal word of the group $G$ if it follows from $X=Y$ in $G$ that $|X| \leqslant|Y|$, where $|Z|$ denotes the length of the word $Z$. Let $W$ be the set of all non-empty words over the alphabet $\Omega$ written in the normal form, that is, every element $X$ in $W$ is written in the form $X_{1} \ldots X_{k}$, where each $X_{l}, 1 \leqslant l \leqslant k$, is a nontrivial element of $G_{\mu(l)}, \mu(l) \in I$, and $\mu(l) \neq \mu(l+1)$ for $l=1, \ldots, k-1$. Then a mapping $F: 2^{W} \backslash\{\emptyset\} \rightarrow 2^{\Omega}$ is defined in the following way: if $C \subseteq W$ and $C \neq \emptyset$, then let $V$ be the set of all letters occuring in the expressions of words of $C$. Then we set $F(C)=f(V)$. 
The main result of this paper is the following embedding scheme.

Theorem A. Let $g_{i}: G_{i} \rightarrow H$ be a set of arbitrary homomorphisms of the groups $G_{i}$ into a group $H$ with kernels $N_{i}, i \in I$, such that a system of subgroups $\left\{g_{i}\left(G_{i}\right)\right\}_{i \in I}$ generates $H$, let $\left\{N_{j}\right\}_{j \in I_{1}}, I_{1} \subseteq I$, be the set of nontrivial groups of the set $\left\{N_{i}\right\}_{i \in I}, \Omega_{1}^{1}$ the free amalgam of the groups $N_{j}, j \in I_{1}$, and also let $f$ be an arbitrary generating mapping on $\Omega$ such that $f(C) \cap \Omega_{1}^{1} \neq \emptyset$ if $C \nsubseteq G_{i}$ for each $i \in I$ and $C$ is non-dihedral. If the set $\left\{N_{j}\right\}_{j \in I_{1}}$ contains either three groups or two groups of which one has order at least 3 , then the free amalgam $\Omega^{1}$ of the groups $G_{i}$ can be embedded in an aspherical atoroidal group $G=g p\{\Omega\}$ with the following properties:

1) the free amalgam $\Omega_{1}^{1}$ is embedded in a normal simple infinite subgroup $L$ of $G$ such that $G / L \cong H$;

2) if $X \in G$ and $X$ is not conjugate in $G$ to an element of one of the groups $G_{i}, i \in I$, then $X$ is of infinite order;

3) every subgroup $M$ of $G$ is either a cyclic group or infinite dihedral (if one of the groups $G_{i}, i \in I$, has involutions), or $M \cap L=1$ and the homomorphic image of $M$ in $H \cong G / L$ has an element of infinite order, or if $M$ is not cyclic or infinite dihedral, then $M$ is conjugate in $G$ to an extension $G_{C, H^{\prime}}$ of a group $H^{\prime}$ by a normal subgroup $L_{C}$ (that is, $G_{C, H^{\prime}} / L_{C} \cong H^{\prime}$ ), where $H^{\prime} \leqslant H$ and $L_{C} \leqslant L$. In what follows, using the notation $L_{C}$, we assume that every element of $L_{C}$ is a minimal word of $G$, and $C=F\left(L_{C} \backslash\{1\}\right)$ or $C=\emptyset$ in the case $L_{C}=\{1\}$;

4) $L_{C}=R_{C} \cap L$, where $R_{C}=g p\{C\}$ if $C \in 2^{\Omega} \backslash\{\emptyset\}$ or $R_{C}=\{1\}$ in the case $C=\emptyset$, and if $C \nsubseteq G_{i}$ for each $i \in I$, then $G_{C, H^{\prime}} \leqslant R_{C}, L_{C}$ is a simple group, $N_{G}\left(L_{C}\right)=R_{C}$ and $C_{G}\left(L_{C}\right)=\{1\}$;

5) if $C \nsubseteq G_{i}$ for each $i \in I$, then Aut $L_{C} \cong R_{C}$ and Out $L_{C} \cong R_{C} / L_{C}$ (in particular, Aut $L \cong G$ and Out $L \cong H$ ), and if $g \in\left(G_{i} \cap C\right) \backslash \Omega_{1}^{1}, i \in I$, then the mapping $g: L_{C} \rightarrow g^{-1} L_{C} g$ is a regular automorphism of $L_{C}$ (that is, $g(a)=a$ if and only if $a=1$ ) if and only if there is no $c \in G_{i} \cap C \cap \Omega_{1}$, where $\Omega_{1}=\Omega_{1}^{1} \backslash\{1\}$, such that $[g, c]=1$;

6) if $C \nsubseteq G_{i}$ for each $i \in I$, then for each $a \in C \cap \Omega_{1}$, we have that $L_{C}=$ $g p\left\{c b a b^{-1} c^{-1} ; b, c \in C\right\}$ (in particular, $L=g p\left\{c b a b^{-1} c^{-1} ; b, c \in \Omega\right\}$, where $a$ is an arbitrary element of $\Omega_{1}$ );

7) if $X$ is a minimal nontrivial word of the group $G$, then $X \in R_{C}$ if and only if $F(\{X\}) \subseteq f(C)$;

8) if $\left\{G_{j}\right\}_{j \in J}, J \subseteq I$, is a set of all groups having nontrivial intersections with a subgroup $R_{C}$ of $G$ and $X \in Z^{-1} R_{C} Z$, where $Z$ is of minimal length among all words in $R_{C} Z$ and $G_{j} Z$, then $F(\{Z\}) \subseteq F(\{X\})$;

9) if $C \nsubseteq G_{i}$ for each $i \in I$ and $M$ is a subgroup of $G$ in which every element is a minimal word in $G$, then $g p\left\{L_{C}, M\right\} \cap L=L_{C_{1}}$, where $C_{1}=F(C \cup(M \backslash\{1\}))$; 
10) if $N_{s}=\{1\}$ for some $s \in I$ and the homomorphism $g_{j}: G_{j} \rightarrow H$ is trivial for each $j \in I \backslash\{s\}$, then $G$ is the semidirect product of $B$ and $L$;

11) if a subgroup $M$ of $G$ is contained in some group $G_{i}, i \in I$, then $N_{G}(M)=$ $N_{G_{i}}(M)$ and $C_{G}(M)=C_{G_{i}}(M)$;

12) if a subgroup $M$ of $G$ is infinite dihedral and not conjugate in $G$ to a subgroup of some group $G_{i}, i \in I$, then $N_{G}(M)$ is infinite dihedral and $C_{G}(M)=\{1\}$;

13) if a cyclic subgroup $M=g p\{A\}$ of $G$ is not conjugate in $G$ to a subgroup of some group $G_{i}, i \in I$, and $A$ is not a product of two involutions in $G$, then $N_{G}(M)$ is cyclic and $N_{G}(M)=C_{G}(M)$.

Now we have the following strengthenings of Theorems B and D [6].

TheOREM B. Let $\left\{G_{i}\right\}_{i \in I}$ be an arbitrary set of nontrivial groups containing either three groups or two groups of which one has order at least $3, H$ an arbitrary (for example, trivial) group, $\Omega^{1}$ the free amalgam of the groups $H$ and $G_{i}, i \in I$, and let $f$ be an arbitrary generating mapping on $\Omega=\Omega^{1} \backslash\{1\}$. Then the free amalgam $\Omega^{1}$ can be embedded in an aspherical atoroidal group $G=g p\{\Omega\}$ such that

1) the free amalgam of the groups $G_{i}$ is embedded in a simple normal infinite subgroup $L$ of $G$ and $G$ is the semidirect product of $H$ and $L$;

2) every nontrivial subgroup of $L$ is infinite cyclic or infinite dihedral (if one of the groups $G_{i}, i \in I$, or $H$ has involutions), or conjugate in $G$ to a subgroup $L_{C}=R_{C} \cap L$, where $R_{C}=g p\{C\}, C \in 2^{\Omega} \backslash 2^{H}$, and if $C \nsubseteq G_{i}$ for each $i \in I$, then $L_{C}$ is simple and $L_{C}=g p\left\{c b a b^{-1} c^{-1} ; b, c \in f(C)\right\}$ for each $a \in f(C) \backslash H$;

3) if $C \nsubseteq G_{i}$ for each $i \in I$, then Aut $L_{C} \cong R_{C}$ and Out $L_{C} \cong R_{C} / L_{C}$ (in particular, Aut $L \cong G$ and Out $L \cong H$ ), and for each $g \in H \cap C, g$ is a regular automorphism of $L_{C}$.

PRoOF: Let $g_{i}: G_{i} \rightarrow H$ be the trivial homomorphism for each $i \in I, g_{H}$ : $H \rightarrow H$ the natural isomorphism. Then the system $\left\{N_{i}\right\}_{i \in I}$ of nontrivial kernels of the homomorphisms $g_{H}$ and $g_{i}, i \in I$, is the same as the set of the groups $G_{i}, i \in I$, and hence Theorem A applies to $\Omega^{1}$ and $f$ and yields the required $G$.

For countable groups we have the following important result.

THEOREM C. Let $\left\{G_{i}\right\}_{i \in I}$ be a countable set of nontrivial countable groups containing either three groups or two groups of which one has order at least $3, H$ an arbitrary countable (for example, trivial) group. Then the free amalgam $\Omega^{1}$ of the groups $H$ and $G_{i}, i \in I$, can be embedded in a group $G=g p\{\Omega\}$, where $\Omega=\Omega^{1} \backslash\{1\}$, with the following properties:

1) the free amalgam of the groups $G_{i}$ is embedded in a simple normal infinite subgroup $L=g p\{\Omega \backslash H\}$ of $G$ and $G$ is the semidirect product of $H$ and $L$;

2) Aut $L \cong G$ (and Out $L \cong H$ ) and for each $g \in H \backslash\{1\}, g$ is a regular automor- 
phism of $L$;

3) if $X, Y \in L$ with $X \in G_{i} \backslash\{1\}, Y \notin G_{i}$ for some $i \in I$, then either $L$ is generated by the pair $(X, Y)$ or $X$ and $Y$ are involutions, or $X$ and $X Y$ are involutions in $G$;

4) every proper subgroup of $L$ is either infinite cyclic or infinite dihedral (if one of the groups $G_{i}, i \in I$, or $H$ has involutions), or contained in a subgroup conjugate in $G$ to some $G_{i}, i \in I$.

Proof: We define a generating mapping $f$ on $\Omega$ in the following way: if $C \subseteq \Omega$ such that $C \nsubseteq G_{i}$ for each $i \in I, C \nsubseteq H$ and $C$ is not dihedral (it follows from the statement of the theorem that such a subset $C$ exists), then $f(C)=\Omega$. Then Theorem B applies to $\Omega^{1}$ and this mapping $f$ and yields the group $G$ satisfying properties 1,2 and 4 in the statement of the theorem. Assertion 3 of the theorem can be proved in the same way as in [ 7 , Theorem 2].

The last application of Theorem A is devoted to construction of a "universal" uncountable group. It is easy to see that there is no countable groups containing every countable group, since any countable group has a countable set of finitely generated subgroups, but by [2], there exists a continuum of pairwise non-isomorphic finitely generated groups. On the other hand, Shelah [9] constructed an uncountable group with all proper subgroups countable, and the existence of such a group with some additional properties (such as Artinian and of finite exponent) follows immediately from [3, Corollary 5]. But there were no examples of uncountable groups $G$ with all proper subgroups countable such that every countable group is contained in $G$. It is obvious that such groups can not be obtained without assuming CH (that is, $2^{\aleph_{0}}=\aleph_{1}$ ).

TheOREM D. Let $H$ be an arbitrary group with $1 \leqslant|H| \leqslant 2^{\aleph_{0}}$. Then assuming $C H$, there exists a simple uncountable group $L$ in which all proper subgroups are countable such that $L$ contains every countable group and Out $L \cong H$.

ProOF: Let $\left\{G_{i}\right\}_{i \in I}$ be the set of all pairwise non-isomorphic countable groups, $\Omega_{1}^{1}=\left\{a_{j} ; 1 \leqslant j<\omega_{1}\right\} \cup\{1\}$ the free amalgam of the groups $G_{i}, i \in I$, and also let $H=\left\{h_{s} ; 1 \leqslant s<\chi\right\} \cup\{1\}$ for some ordinal number $\chi \leqslant \omega_{1}$ or $H=\{1\}$, where $\omega_{1}$ is the first uncountable ordinal number. A generating mapping $f$ on $\Omega=\left(\Omega_{1}^{1} \cup H\right) \backslash\{1\}$ is defined in the following way: if $C$ is a finite non-dihedral subset of $\Omega$ such that $C \nsubseteq G_{i}$ for each $i \in I$ and $C \nsubseteq H$, then let $\mu$ be the maximal ordinal number such that either $a_{\mu}$ or $h_{\mu}$ is contained in $C$, and we set

$$
f(C)=f(\Omega(\mu) \cap H) \cup \bigcup_{i \in I} f\left(\Omega(\mu) \cap G_{i}\right)
$$

where $\Omega(\mu)=\left\{a_{j} ; 1 \leqslant j \leqslant \mu\right\} \cup\left\{h_{s} ; 1 \leqslant s \leqslant \mu\right\}\left(\Omega(\mu)=\left\{a_{j} ; 1 \leqslant j \leqslant \mu\right\}\right.$ in the case 
$H=\{1\})$. It is easy to see that this mapping $f$ satisfies all conditions in the definition of a generating mapping on $\Omega$.

Theorem B applied to $\Omega \cup\{1\}$ and $f$ and yields a group $G$ with a simple uncountable normal subgroup $L$ such that every countable group is contained in $L$ and Out $L \cong H$. Let $M$ be a non-cyclic and non-dihedral proper subgroup of $L$. Then by Theorem $\mathrm{B}, M$ is conjugate in $G$ to a subgroup $L_{C}=R_{C} \cap L$, where $R_{C}=g p\{C\}, C \in 2^{\Omega} \backslash 2^{H}$. We may assume that $C \nsubseteq G_{i}$ for each $i \in I$, hence the set $C$ is countable, since otherwise it follows from the definition of the mapping $f$ that $f(C)=\Omega$ and $M=L$, and we arrive at a contradiction to the choice of $M$. Therefore, the subgroup $M$ is countable, which completes the proof of the theorem.

The proof of Theorem A will be heavily based on the results from [6] and [7]. Unless otherwise stated, all definitions and notation may be found in [7] and [8].

\section{Construction of the Group G}

As in [8], we introduce the positive parameters

$$
\alpha, \beta, \gamma, \delta, \varepsilon, \zeta, \eta, \iota
$$

where all the parameters are arranged according to "height", that is, each constant is chosen after its predecessor. Our proofs and some definitions are based on a system of inequalities involving these parameters. The value of the parameters can be chosen in such a way that all the inequalities hold. We then use the following notation:

$$
\alpha^{\prime}=1 / 2+\alpha, \beta^{\prime}=1-\beta, \gamma^{\prime}=1-\gamma, h=\delta^{-1}, d=\eta^{-1}, n=\iota^{-1} .
$$

We may assume that $n$ is an integer. We also use the notation introduced in Section 1 .

We may assume that $I$ is a well-ordered set. We also may assume that $\Omega^{1}$ is a well-ordered set such that 1 is the maximal element of $\Omega^{1}$ and if $a \in G_{i} \backslash\{1\}$ and $b \in G_{j} \backslash\{1\}$, where $i<j$, then $a<b$. On the set $\Omega_{2}=\left\{a b \mid a \in \Omega^{1}, b \in \Omega\right.$ and if $\{a, b\} \subseteq G_{i}$ for some $i \in I$, then $\left.a=1\right\}$ we introduce an order in the following way: $a b \leqslant c d$ if and only if either $b<d$ or $b=d$ and $a \leqslant c$ (with respect to the ordering of $\left.\Omega^{1}\right)$.

By the statement of Theorem $\mathrm{A}$, there is a homomorphism of the free product $G(1)$ of the groups $G_{i}, i \in I$, onto $H$ such that its restriction to every group $G_{i}$ is equal to $g_{i}$. Suppose that the kernel of this homomorphism is $N$.

Let $D_{1}=\emptyset$, and suppose, by induction, that we have defined the set of relators $D_{i-1} \subseteq N, i \geqslant 2$, and set

$$
G(i-1)=\left\langle G(1) \| R=1 ; R \in D_{i-1}\right\rangle .
$$


A word $X$ is called free in rank $i-1$ if $X$ is not conjugate in rank $i-1$ to an element of $\Omega^{1}$, that is, to an image in $G(i-1)$ of an element of one of the free factors $G_{j}$. A non-empty word $Y$ is said to be simple in rank $i-1$ if it is free in rank $i-1$, not conjugate in rank $i-1$ (that is, in $G(i-1)$ ) to a power of a shorter word and not conjugate in rank $i-1$ to a power of a period of rank $k<i$.

Now let $P_{i}$ denote a maximal set of words of length $i$ which are simple in rank $i-1$ with the property that $A, B \in P_{i}$ and $A \not \equiv B$ ("三" means letter-for-letter equality of words of the same length) implies that $A$ is not conjugate in rank $i-1$ to $B$ or $B^{-1}$. The words in $P_{i}$ are called periods of rank $i$. A special role in the construction of the group $G$ will be played by the sets $P_{i}^{\prime}$ of all periods of rank $i$ which are not equal in rank $i-1$ to a product of two involutions (of $G(i-1)$ ). (For short, a product of two involutions of a group will be called a dihedral element of a group.) We may assume (see Lemma 3.1 below) that if $a, c \in \Omega_{1}, b, e, g \in \Omega$ and $d, f \in \Omega^{1}$ such that $a$ is of infinite order (if such an $a$ exists), $\{a, b\} \nsubseteq G_{i},\{c, e\} \nsubseteq G_{j}$ and $\{c, g\} \nsubseteq G_{s}$ for each $i, j, s \in I, f g, d e \in \Omega_{2}, f g \neq d e$ and $f g e^{-1} d^{-1} \neq c$ in the case $c^{2}=1$, then the words $A_{0}=a[a, b]^{k} a[a, b]^{2 k} a[a, b]^{3 k}, A_{m}=a A_{0}^{m}, B_{0}=(c f g)^{-1}\left[[c, d e]^{n},[c, f g]\right](c f g), B_{m}=$ $(c f g)^{-1}[c, d e]^{n}(c f g) B_{0}^{m}$ are non-dihedral periods of some ranks for each $k \geqslant 1$ and $m,|m|>n^{6}$.

For each period $A \in P_{i}^{\prime} \cap N$, we fix a maximal subset $Y_{A}$ such that:

1) if $T \in Y_{A}$, then $1 \leqslant|T|<d|A|$;

2) each double coset of the pair $g p\{A\}, g p\{A\}$ of subgroups of $G(i)$ contains at most one word in $Y_{A}$ and this word is of minimal length among the words representing this double coset;

3) if $T \in Y_{A}$, then $T \in N$ and $F(\{T\}) \subseteq F(\{A\})$.

We may assume (see Lemma 3.1 below) that if a power $F^{t}$ of a period $F$ of some rank is conjugate to a word $B C^{m}$ for some $m \geqslant n$, where $C$ is a non-dihedral period of rank $i$ not equal to $A_{0}$ or $B_{0},|B|<\iota(m|C|)^{1 / 3}$ and $B C B^{-1} \neq C^{ \pm 1}$ in $G(i-1)$, then $t=1$.

For each period $A \in P_{i}^{\prime} \cap N$, we introduce the ordering of the set of natural numbers (or a finite segment of it) on the set $Y_{A}$ such that the first element of the set $Y_{A}$ belongs to $\Omega_{1}$ (it follows from the statement of Theorem A that $Y_{A} \cap \Omega_{1} \neq 0$ ) and if $A=A_{m}$ or $A=B_{m}$, where $m=0$ or $|m|>n^{6}$, then the first element of the set $Y_{A}$ is $a$ or $\min (c, h)$ (with respect to the ordering of $\Omega^{1}$ ), respectively, where $h=d$ if $d \in \Omega_{1}$, otherwise $h=1$. We denote this order by $\leqslant_{A}$.

For each period $A \in P_{i}^{\prime} \cap N, i \geqslant 7$, we now define some relations. If $A=A_{m},|m|>$ $n^{6}$, for some $a \in \Omega_{1}$ and $b \in \Omega$ such that $\{a, b\} \nsubseteq G_{i}$ for each $i \in I$ and $a$ is of infinite order, then for each $k, 5 \leqslant k \leqslant 15$, we introduce a relation

$$
a^{-1} A^{n} a A^{n+k} a A^{n+30+k} \ldots a A^{n+30(h-2)+k}=1 \text {. }
$$


If $A=B_{m},|m|>n^{6}$, for some $c \in \Omega_{1}, e, g \in \Omega$ and $d, f \in \Omega^{1}$ such that $\{c, e\} \nsubseteq$ $G_{i},\{c, g\} \nsubseteq G_{j}$ for each $i, j \in I, f g, d e \in \Omega_{2}, f g \neq d e$ and $f g e^{-1} d^{-1} \neq c$ in the case $c^{2}=1$, then for each $k, 16 \leqslant k \leqslant 25$, and $T=(c f g)^{-1}[c, d e]^{n}(c f g)$, we consider the relation

$$
T^{-1} A^{n} T A^{n+k} T A^{n+30+k} \ldots T A^{n+30(h-2)+k}=1,
$$

and if $b_{1}=\min (c, e), b_{2}=\min (d e, f g)$ (with respect to the ordering of $\Omega_{2}$ ) and $T_{i}=(c f g)^{-1}[c, d e]^{i}(c f g), i \in\{1,2\}$, then we set

$$
(c f g)^{-1} b_{i} c b_{i}^{-1}(c f g) A^{n} T_{i} A^{n+25} T_{i} A^{n+55} \ldots T_{i} A^{n+30(h-2)+25}=1
$$

for each $i, 1 \leqslant i \leqslant 2$. Let $T \in Y_{A}$ and $T \neq a$ in the case $A=A_{m},|m|>n^{6}$. If $a$ is the minimal element of the set $Y_{A}$ and $T \neq a$, then we introduce the relation

$$
a A^{n} T A^{n+10} T A^{n+40} \ldots T A^{n+30(h-2)+10}=1,
$$

and if $T=a$, then it follows from the definition of the set $P_{i}$ that there exists $b \in$ $F(\{A\})$ such that $\{a, b\} \nsubseteq G_{i}$ for each $i \in I$, and we consider the relation

$$
{ }_{b a b}{ }^{-1} A^{n} T A^{n+10} T A^{n+40} \ldots T A^{n+30(h-2)+10}=1 .
$$

If $a$ is the first element of the set $Y_{A}, T \in Y_{A} \backslash\{a\}$ and $T \neq(c f g)^{-1}[c, d e]^{n}(c f g)$ in the case $A=B_{m},|m|>n^{6}$, then we introduce a relation

$$
a A^{n} T A^{n+20} T A^{n+50} \ldots T A^{n+30(h-2)+20}=1,
$$

and if $T=a$, then, as above, we set

$$
b a b^{-1} A^{n} T A^{n+20} T A^{n+50} \ldots T A^{n+30(h-2)+20}=1
$$

for some $b \in F(\{A\})$ such that $\{a, b\} \nsubseteq G_{i}$ for each $i \in I$. And if $T \in Y_{A}$, then let $T_{1}$ be the minimal element of the set $Y_{A} \backslash\left\{a^{ \pm 1}\right\}$ such that $T<_{A} T_{1}$ (if such an element $T_{1}$ exists). Then we consider the relation

$$
T_{1} A^{n} T A^{n+30} T A^{n+60} \ldots T A^{n+30(h-1)}=1 .
$$

The left-hand sides of the relations (2.1)-(2.8) form the set $S_{i}$ of relators of rank $i$. For each $i \geqslant 2$, we set $D_{i}=D_{i-1} \cup S_{i}$, and the group $G(i)$ is defined by its presentation:

$$
G(i)=\left\langle G(1) \| R=1 ; R \in D_{i}\right\rangle
$$

Finally, we define

$$
G=\left\langle G(1) \| R=1 ; R \in D=\bigcup_{i \geqslant 1} D_{i}\right\rangle .
$$

By a diagram of rank $i$, where $i \geqslant 2$, we mean a diagram over the presentation (2.9). Contours of cells $I I$ in the diagrams under considerations split to words of the form (2.1)-(2.8). Those sections of $\Pi$ with labels $\left(A^{n+s}\right)^{ \pm 1}$ are called long sections while the others are called short sections of the contour. 


\section{Proof of Theorem A}

We start our proof of the theorem with

LEMMA 3.1. The choice of the set of periods of the group $G$ is correct.

Proof: Let $a, c \in \Omega_{1}, b, e, g \in \Omega$ and $d, f \in \Omega^{1}$ such that $a$ is of infinite order (if such an $a$ exists), $\{a, b\} \nsubseteq G_{i},\{c, e\} \nsubseteq G_{j}$ and $\{c, g\} \nsubseteq G_{\text {s }}$ for each $i, j, s \in I$, $f g, d e \in \Omega_{2}, f g \neq d e$ and $f g e^{-1} d^{-1} \neq c$ in the case $c^{2}=1$. Also let $C=A_{0}=$ $a[a, b]^{k} a[a, b]^{2 k} a[a, b]^{3 k}$ for some $k \geqslant 1$ or $C=B_{0}=(c f g)^{-1}\left[[c, d e]^{n},[c, f g]\right](c f g)$, and suppose that $C$ is conjugate in some rank $i \geqslant 1$ to $V$, where $V$ is either an element of $\Omega$ or a power of a period of rank $\leqslant i$, or a power of a simple word in rank $i$. Then it follows from [8, Lemma 26.5], [8, Corollary 22.2], [8, Lemma 21.7] and the definition of the relations in $G$ that $C$ and $V$ are also conjugate in $G(1)$. Hence by the choice of $C$, we may assume that $C$ is a period of some rank. Moreover, if $C$ is a dihedral element in $G$, then there is $X \in G$ such that $X C X^{-1}=C^{-1}$ in some rank $i$. Then as above, we may assume that $i=0$, and we arrive at a contradiction to the choice of $C$. Thus we may assume that $A_{0}$ and $B_{0}$ are non-dihedral periods.

If $C_{1}$ is a non-dihedral period of some rank $i$ and $B_{1}$ is a word such that $B_{1} C_{1} B_{1}^{-1} \neq C_{1}^{ \pm 1}$ in rank $i-1$ and $\left|B_{1}\right|<\iota\left(|m|\left|C_{1}\right|\right)^{1 / 3}$ for some $m$ such that $|m| \geqslant n$, then by the proof of [7, Lemma 7] and [8, Lemma 34.7], a word $B_{1} C_{1}^{m}$ is conjugate in $G$ to a power $F^{t}$ of a non-dihedral period $F$ of some rank. Repeating the proof of [8, Lemma 27.3] with a reference to [8, Lemma 23.15] replaced by a reference to [7, Lemma 8], we obtain that $|t|=1$. (In particular, it is true for $A_{m}$ and $B_{m}$, where $|m|>n^{6}$.)

If $F^{-t}$ is conjugate to a product $B_{2} C_{2}^{m_{2}}$ of the same type, then there is a reduced annular diagram $\Delta$ with contours $z_{1} p_{1}$ and $z_{2} p_{2}$, where $\phi\left(z_{i}\right) \equiv B_{i}$ and $\phi\left(p_{i}\right) \equiv$ $C_{i}^{m_{i}}, i=1,2$. Repeating the argument of [7, Lemma 8], we have that there exists a contiguity submap $\Gamma$ of $p_{1}$ to $p_{2}$ such that the sum of lengths of its contiguity arcs is greater than $\beta^{\prime}\left(\left|p_{1}\right|+\left|p_{2}\right|\right)$. Then by [8, Lemma 25.10] (with the correction from [7]), we have that $C_{1} \equiv C_{2}$ and either $m_{1} m_{2}>0, p_{1}$ and $p_{2}$ are $C_{1}$-anticompatible in $\Delta$ and $C_{1}$ is dihedral, which contradicts the choice of the words $C_{1}$ and $C_{2}$, or $m_{1} m_{2}<0$ and $p_{1}$ and $p_{2}$ are $C_{1}$-compatible in $\Delta$. In the second case, we have that $B_{1} \in g p\left\{C_{1}\right\} B_{2}^{-1} g p\left\{C_{1}\right\}$, and in order to complete the proof of the lemma, it remains to consider the cases when either 1) $B_{1} \equiv B_{2} \equiv a$ and $C_{1} \equiv C_{2} \equiv A_{0}$ for some $a \in \Omega_{1}$ and $b \in \Omega$ such that $\{a, b\} \nsubseteq G_{i}$ for each $i \in I$ and $a$ is of infinite order in $G$, or 2) $B_{1} \equiv B_{2} \equiv(c f g)^{-1}[c, d e]^{n}(c f g)$ and $C_{1} \equiv C_{2} \equiv B_{0}$ for some $c \in \Omega_{1}, e, g \in \Omega$ and $d, f \in \Omega^{1}$ such that $\{c, e\} \nsubseteq G_{j},\{c, g\} \nsubseteq G_{s}$ for each $j, s \in I, f g, d e \in \Omega_{2}, f g \neq d e$ and $f g e^{-1} d^{-1} \neq c$ in the case $c^{2}=1$.

It follows from the proof of $\left[8\right.$, Lemma 25.18] that in any case, either $B_{1} \in g p\left\{C_{1}\right\}$, 
which is impossible, or $\left(C_{1}^{s} B_{1}\right)^{2}=1$ in $G$ for some integer $s$. We note that $s \neq 0$, since $B_{1}$ is of infinite order in $G$. Then by [8, Lemma 34.7], $C_{1}^{s} B_{1}$ is conjugate in $G$ to an element $v$ of $\Omega$, and it follows from [8, Corollary 22.2] and [8, Lemma 21.7] that $C_{1}^{s} B_{1}$ and $v$ are conjugate in $G(1)$. We arrive at a contradiction to the choice of the words $C_{1}$ and $B_{1}$. Thus we may assume that $t=1$, and the proof of the lemma is complete.

Immediate verification shows that the presentations (2.9) of the groups $G(i)$ satisfy condition $R$ (see [8, Sections 25 and 34]). So we can apply to the diagrams under considerations all the results from [7] and also [6, Lemmas 1-4] if in the definition (from [6]) of an $I$-diagram we demand that condition I 3 holds for all contiguity submaps of $q_{i_{1}}^{0}$ to $q_{i_{2}}^{0}$, where $i_{1}, i_{2} \in\{1,2\}$. We also need the following analogue of [6, Lemma 5].

Lemma 3.2. Let $C$ be a period of the group $G, k$ an integer such that $|k|>100 \zeta^{-1}$, and also let $W$ be a word which does not commute with $C^{k}$ in $G$ and whose length is minimal among all words in the double coset $g p\left\{C^{k}\right\} W g p\left\{C^{k}\right\}$. Then $\left[C^{k}, W\right]=Z A^{l} Z^{-1}$ in $G$, where $A$ is a period and $Z$ is a minimal word in $G$, and

$$
F(\{A\})=F(\{C, W\}), F(\{Z\}) \subseteq F(\{A\}) .
$$

Moreover, if $W C^{k} W^{-1} \neq C^{ \pm k}$ in $G$, then also $B A^{l} B^{-1} \neq A^{ \pm l}$ in $G$ for $B=Z^{-1} C^{k} Z$.

Proof: By [8, Lemma 34.7], the word $\left[C^{k}, W\right]$ is conjugate in $G$ to a word $V$, where either $|V|=1$ or $V=A^{l}$ for some period $A$ and an integer $l$. Let $\Delta$ be a reduced annular diagram with contours $p$ and $q$ such that $\phi(q) \equiv V^{-1}, p=p_{1} p_{2} p_{3} p_{4}, \phi\left(p_{1}\right) \equiv$ $\phi\left(p_{3}^{-1}\right) \equiv C^{k}, \phi\left(p_{2}\right) \equiv \phi\left(p_{4}^{-1}\right) \equiv W$. By pasting together the paths $p_{2}$ and $p_{4}^{-1}$, we obtain a diagram $\Delta^{\prime}$ on a sphere with three holes whose reduced form (that is, with $j$-pairs removed) is denoted by $\Delta_{0}$. The cyclic sections $p_{1}$ and $p_{3}$ can be assumed smooth in $\Delta_{0}$.

We note that there is no contiguity submap $\Gamma$ of $p_{i_{1}}$ to $p_{i_{2}}$, where $i_{1}, i_{2} \in\{1,3\}$ and $i_{1} \neq i_{2}$, such that $\left(p_{i_{1}}, \Gamma, p_{i_{2}}\right) \geqslant 1 / 100$, since otherwise it follows from [8, Lemma 25.10] that $p_{i_{1}}$ and $p_{i_{2}}$ are $C$-compatible in $\Delta_{0}$, and using [8, Lemma 24.9], we arrive at a contradiction to the choice of the word $W$. Suppose now that for some $i \in\{1,3\}$, there is a contiguity submap $\Gamma$ of $p_{i}$ to $p_{i}$ such that $\left(p_{i}, \Gamma, p_{i}\right) \geqslant 1 / 100$. Then by [8, Lemma 25.8] (with the correction from [7]), $p_{i}$ is $C$-anticompatible, and for a compatible path $t$ (see the definition of $C$-anticompatibility from [7]) and a word $X$ we have that $C=X \phi(t)$ in $G$, where $X$ and $\phi(t)$ are involutions in $G$. Hence there exists an annular subdiagram $\Delta_{0}^{\prime}$ of $\Delta_{0}$ with contours $p_{i}^{\prime} t^{\epsilon}$ and $p_{4-i}$, where $p_{i}^{\prime}$ is a subpath of $p_{i}$ with label equal to a power of $C$ and $|\varepsilon|=1$. Therefore, a power of $C$ is conjugate in $G$ to an involution, which contradicts [8, Lemma 34.7]. Thus we obtain that $\Delta_{0}$ satisfies conditions I1-I3 from the definition (from [6]) of an $I$-diagram. 
If $|V|=1$, then as in the proof of [8, Lemma 24.6], we obtain that there is a contiguity submap $\Gamma$ of $p_{i_{1}}$ to $p_{i_{2}}$ for some $i_{1}, i_{2} \in\{1,3\}$ such that $\left(p_{i_{1}}, \Gamma, p_{i_{2}}\right)>1 / 10$, and we arrive at a contradiction to I3. Thus $V=A^{l}$ in $G$ for some period $A$ and an integer $l$.

The second assertion of the lemma can be proved in the same way as in $[6$, Lemma $5]$.

In order to prove the last assertion of the lemma, we note that the inequality $B A^{l} B^{-1} \neq A^{ \pm l}$ is equivalent to the inequality $C^{k}\left[C^{k}, W\right] C^{-k} \neq\left[C^{k}, W\right]^{ \pm 1}$ which is true, since otherwise we have that either $C^{k}\left(W C^{-k} W^{-1}\right)=\left(W C^{-k} W^{-1}\right) C^{k}$ or $C^{2 k} W=W C^{2 k}$ in $G$, and it follows from [8, Lemma 34.9] and [8, Lemma 25.15] (with the correction from [7]) that $W C^{k} W^{-1}=C^{ \pm k}$, which contradicts our assumption about $W$.

The proof of the lemma is complete.

By an $H$-map we understand a circular or annular $B$-map $\Delta$ with contours $p$ (in the case of a circular map) or $p$ and $q$ (in the annular case), where $p=s_{1} t_{1} \ldots s_{l} t_{l}, l \leqslant$ $3, s_{1}, \ldots, s_{l}$ are called long sections of the first kind, $t_{1}, \ldots, t_{l}$ short sections, and $q$ a long section of the second kind; all sections are assumed (cyclically) reduced and, for some $j>1$, the following conditions hold.

H1. Every section of the first kind is a smooth section of rank $j$ and $\left|s_{1}\right| \geqslant n j$.

H2. The section $q$ of the second kind is either smooth or geodesic.

H3. The short sections are geodesic and the length of any short section is less than $\max \left(d j, \iota\left|s_{1}\right|^{1 / 3}\right)$.

Lemma 3.3. The assertion of [8, Lemma 23.15] for $C$-maps is also true for $H$ maps.

Proof: The lemma can be proved in the same way as [8, Lemma 23.15] taking into account the remark made in the proof of [7, Lemma 5].

LEMma 3.4. If $T A^{k} T^{-1}=A^{\text {ek }}$ in $G$ for some period $A$ and an integer $k$, where $|\varepsilon|=1$, then $T A T^{-1}=A^{e}$ in $G$ and either $\varepsilon=1$ and $T \in g p\{A\}$ or $\varepsilon=-1$ and $A$ is a dihedral element of $G$.

Proof: If $\varepsilon=1$, then by [8, Lemma 34.9], $T \in g p\{A\}$. Suppose now that $\varepsilon=-1$. Then $A^{k m} T^{-1} A^{k m} T=1$ in $G$, where $m$ is chosen in such a way that $m \geqslant n$ and $|T|<\iota(m|A|)^{1 / 3}$, and let $\Delta$ be a reduced circular diagram (of some rank) with contour $s_{1} t_{1} s_{2} t_{2}$, where $\phi\left(t_{1}\right) \equiv \phi\left(t_{2}^{-1}\right) \equiv T^{-1}$ and $\phi\left(s_{1}\right) \equiv \phi\left(s_{2}\right) \equiv A^{k m}$. It follows from [8, Lemma 26.5] and the choice of $m$ that $\Delta$ is an $H$-map. Then by Lemma 3.3 , there exists a contiguity submap $\Gamma$ of $s_{1}$ to $s_{2}$ such that $\left|\Gamma \wedge s_{1}\right|>\left|s_{1}\right| / 2$, and it follows from [8, Lemma 25.8] (with the correction from [7]) that $s_{1}$ and $s_{2}$ are $A$ anticompatible in $\Delta$ and $A$ is a dihedral element. Hence by $[7$, Lemma 3$]$, we have 
that $T A T^{-1}=A^{-1}$, which completes the proof of the lemma.

LEMMA 3.5. Let $A$ be a period and $T$ a word such that $T A T^{-1} \neq A^{ \pm 1}$ in $G$ and $F(\{T\}) \subseteq F(\{A\})$, and also let $m$ be an integer with $|T|<\zeta \iota(|m||A|)^{1 / 3}$ and $|m|>n$. Then $A^{m} T A^{2 m} T A^{3 m} T=Z C^{l} Z^{-1}$ and $T_{1} C^{l} T_{1}^{-1} \neq C^{ \pm l}$ in $G$, where $C$ is a non-dihedral period, $Z$ is a minimal word in $G$ and $T_{1}=Z^{-1} A^{m} Z$, and

$$
F(\{A\})=F(\{C\}), F(\{Z\}) \subseteq F(\{A\}) .
$$

Proof: By [7, Lemma 6], it remains to prove only (3.2) and the inequality $T_{1} C^{l} T_{1}^{-1} \neq C^{ \pm l}$.

It follows from [6, Lemma 1] and [6, Lemma 3] and the statement of the lemma that

$$
F(\{Z, C\}) \subseteq F(\{T, A\})=F(\{A\}) .
$$

So it is sufficient to show that $F(\{A\}) \subseteq F(\{C\})$.

Let $\Delta$ be a reduced annular diagram (of some rank) with contours $p$ and $q$, where $\phi(p) \equiv A^{m} T A^{2 m} T A^{3 m} T$ and $\phi(q) \equiv C^{-l}$. It follows from the statement of the lemma and [8, Lemma 26.5] that $\Delta$ is an $H$-map. Among contiguity submaps $\Gamma_{1}, \Gamma_{2}, \ldots$ given by Lemma 3.3, the submaps in which at least one of the contiguity arcs has length greater than $\zeta^{-1}|A|$ are called long while the others are called short.

If $\Gamma$ is a contiguity submap of a long section of the first kind to a distinct long section of the first kind, say of $s_{1}$ to $s_{2}$, such that its connecting line is homotopic in $\Delta$ to a subpath of $p$ not containing $s_{3}$, then by [8, Lemma 26.5], [8, Lemma 21.1] and [8, Lemma 25.8], $\Gamma$ is a short contiguity submap, since otherwise it follows from [8, Lemma 23.17] and [7, Lemma 3] that $T A T^{-1}=A^{-1}$ in rank $|A|-1$, which contradicts the choice of $T$. But by the statement of the lemma, $\left|s_{k}\right| \geqslant\left(\left|s_{1}\right|+\left|s_{2}\right|+\left|s_{3}\right|\right) / 6$ for each $k \in\{1,2,3\}$, then it follows from the definition of $H$-maps and Lemma 3.3 that there exists a contiguity submap $\Gamma_{i}$ of a long section $s_{i}$ to $q$ for each $i \in\{1,2,3\}$ such that $\left|\Gamma_{i} \wedge s_{i}\right|>\left|s_{i}\right| / 2$, and by [6, Lemma 2], $F(\{A\}) \subseteq F(\{C\})$.

The period $C$ is non-dihedral, so if $T_{1} C^{l} T_{1}^{-1}=C^{e l}$, where $|\varepsilon|=1$, then by Lemma 3.4, $\varepsilon=1$ and we have that $A^{m}=\left(A^{m} T A^{2 m} T A^{3 m} T\right) A^{m}\left(A^{m} T A^{2 m} T A^{3 m} T\right)^{-1}$ in $G$. Again it follows from Lemma 3.4 that $A^{2 m} T A^{3 m} T A^{t} T=1$ in $G$ for some integer $t$. Let $\Delta$ be a reduced circular diagram (of some rank) with contour $s_{1} t_{1} s_{2} t_{2} s_{3} t_{3}$, where $\phi\left(s_{1}\right) \equiv A^{2 m}, \phi\left(s_{2}\right) \equiv A^{3 m}, \phi\left(s_{3}\right) \equiv A^{t}, \phi\left(t_{i}\right) \equiv T$ for each $i \in\{1,2,3\}$. It follows from the statement of the lemma and [8, Lemma 26.5] that $\Delta$ is an $H$-map (even if $t=0$ ). Then using [7, Lemma 3 and Lemma 3.3], we obtain that $T A T^{-1}=A^{-1}$ in $G$, which contradicts the choice of $T$.

The proof of the lemma is complete.

LEMMA 3.6. Let $A$ be a non-dihedral period and $T$ a word such that $T A T^{-1} \neq$ $A^{ \pm 1}$ in $G$ and $F(\{T\}) \subseteq F(\{A\})$, and also let $m$ be an integer with $|T|<\iota(|m||A|)^{1 / 3}$ 
and $|m|>n$. Then $T A^{m}=Z C^{\varepsilon} Z^{-1},\left|T_{1}\right|,|Z|<3|C|,\left(\beta^{\prime}\right)^{4} m|A| \leqslant|C|$ and $T_{1} C T_{1}^{-1} \neq C^{ \pm 1}$ in $G$, where $C$ is a non-dihedral period of some rank, $|\varepsilon|=1, Z$ is a minimal word in $G$ and $T_{1}=Z^{-1} T Z$, and

$$
F(\{C\})=F(\{A\}), F(\{Z\}) \subseteq F(\{C\}) .
$$

Proof: The assertion of the lemma follows immediately from the proofs of $[7$, Lemma 9], [6, Lemma 6 and Lemma 3.1].

Now everything is ready to prove an anolog of [6, Lemma 6$]$.

Lemma 3.7. Let $R=g p\left\{C^{k}, W\right\}$, where $C$ is a period, $C^{k} \in N \backslash\{1\}$ and $W$ is a minimal word in $G$ such that $W C^{k} W^{-1} \neq C^{ \pm k}$ in $G$. Then $R$ contains a non-dihedral period $C_{1} \in N$ such that $F\left(\left\{C_{1}\right\}\right)=F(\{C, W\})$ and $n|C|<\left|C_{1}\right|$.

Proof: By [8, Lemma 34.7], $C$ is of infinite order in $G$, so there exists $p>100 \zeta^{-1}$ such that $C^{p} \in N \cap R$. By [6, Lemma 1], we may assume that $W$ has the minimal length among all words in the double coset $g p\left\{C^{p}\right\} W g p\left\{C^{p}\right\}$, and by $[6$, Lemma 3 and Lemma 3.2], $\left[C^{p}, W\right]=Z_{1} A^{l} Z_{1}^{-1}$, where $A$ is a period, $Z_{1}$ is a minimal word in $G$, and for a word $B$ which is minimal in $G$ and equal in $G$ to a word $Z_{1}^{-1} C^{p} Z_{1}$, we have that

$$
F(\{B\})=F\left(\left\{Z_{1}, C\right\}\right) \subseteq F(\{A\}),
$$

$B A^{l} B^{-1} \neq A^{ \pm l}$ and (3.1) holds. It is obvious that $A^{l}, B \in N$ and $B A B^{-1} \neq A^{ \pm 1}$ in $G$.

Let $t$ be an integer such that $|B|<\zeta \iota(l t|A|)^{1 / 3}$ and $l t>n$. Then by Lemma 3.5, $A^{l t} B A^{2 l t} B A^{3 l t} B=Z_{2} V^{f} Z_{2}^{-1}$ and $T V^{f} T^{-1} \neq V^{ \pm f}$ in $G$, where $V$ is a non-dihedral period, $Z_{2}$ and $T=Z_{2}^{-1} A^{l t} Z_{2}$ are minimal words in $G$, and (3.2) holds. It follows from (3.2) and [6, Lemma 3] that

$$
F(\{T\})=F\left(\left\{Z_{2}, A\right\}\right)=F(\{A\})=F(\{V\}) .
$$

Moreover, $V^{f}, T \in N$, since $A^{l}, B \in N$ and $N$ is a normal subgroup, and $T V T^{-1} \neq$ $V^{ \pm 1}$ in $G$.

Now we choose an integer $m$ such that $|T|<\iota(m f|V|)^{1 / 3},\left|Z_{1}\right|,\left|Z_{2}\right|,|C|<$ $\iota^{2} m f|V|$ and $m f>n$. By Lemma 3.6, $T V^{m f}=Z_{3} C_{1}^{e} Z_{3}^{-1}$ and $\left|T_{1}\right|,\left|Z_{3}\right|<3\left|C_{1}\right|$, where $C_{1}$ is a non-dihedral period, $|\varepsilon|=1, Z_{3}$ and $T_{1}=Z_{3}^{-1} T Z_{3}$ are minimal words in $G$, and (3.3) holds. Moreover, it follows from Lemma 3.6 that

$$
\left|Z_{1}\right|,\left|Z_{2}\right|,|C|<\iota^{2}\left(\beta^{\prime}\right)^{-4}\left|C_{1}\right|<\iota\left|C_{1}\right| .
$$

We also have that $C_{1} \in N$, and by (3.1)-(3.3),

$$
F(\{C, W\})=F(\{A\})=F(\{V\})=F\left(\left\{C_{1}\right\}\right) .
$$


The words $C_{1}$ and $T_{1}$ are contained in $R_{1}=Z^{-1} R Z$, where $Z=Z_{1} Z_{2} Z_{3}$. It follows from [6, Lemma 1], Lemma 3.6 and (3.1)-(3.5) that $F\left(\left\{T_{1}\right\}\right), F(\{Z\}) \subseteq F\left(\left\{C_{1}\right\}\right)$ and $|Z|<4\left|C_{1}\right|$. Hence there are $Z^{\prime}, T_{1}^{\prime} \in Y_{C_{1}}$ such that $Z \in g p\left\{C_{1}\right\} Z^{\prime} g p\left\{C_{1}\right\}$ and $T_{1} \in g p\left\{C_{1}\right\} T_{1}^{\prime} g p\left\{C_{1}\right\}$. By the definition of the relation (2.6) for $C_{1}$ and $T_{1}^{\prime}$ (or for $C_{1}$ and $\left(T_{1}^{\prime}\right)^{2}$ if $C_{1}=B_{m},|m|>n^{6}$, and $T_{1}^{\prime}=(c f g)^{-1}[c, d e]^{n}(c f g)$ ), the minimal element $a$ of the set $Y_{C_{1}}$ is contained in $R_{1}$. Now using the defining relation (2.8) for $C_{1}$ and $a$, we obtain that $a_{1} \in R_{1}$, where $a_{1}$ is the minimal element of the set $Y_{C_{1}} \backslash\left\{a^{ \pm 1}\right\}$, and so on. Thus we have that $Z^{\prime}$ is contained in $R_{1}$, hence $Z \in R_{1}$ and $R=R_{1}$, which completes the proof of the lemma.

Now we may obtain all the assertions of Theorem A, except assertion 5 about the automorphism groups of the groups $L_{C}$, where $C \nsubseteq G_{i}$ for each $i \in I$, if we repeat the proof of [6, Theorem A] replacing references to [6, Lemma 5 and Lemma 6], by references to Lemmas 3.2 and 3.7. We also need to make the following amendments.

1) In order to prove that the homomorphic image $L$ of the subgroup $N$ is an infinite subgroup of $G$, we can use the argument in the proof of [8, Theorem 26.1], with [8, Lemma 34.1] used in place of [8, Theorem 4.6].

2) Let $M$ be an arbitrary non-cyclic subgroup of $G$ containing a free element $X$. (An element $X$ is called free in $G$ if it is free in rank $i$ for each $i \geqslant 1$.) By [8, Lemma 34.7], $X$ is conjugate in $G$ to a power of a period $A$. If $M \cap L=1$, then the image $A$ in $H$ has infinite order, since by [8, Lemma 34.7], $A$ is of infinite order in $G$. In the opposite case, as in the proof of $[6$, Theorem A], we obtain that $M$ is conjugate in $G$ to a subgroup $M_{2}=g p\left\{C^{l},\left\{W_{j}\right\}_{j \in J}\right\}$, where $C$ is a period, $C^{l} \in L$ and for each $j \in J, W_{j}$ is a minimal word in $G$ such that $W_{j}$ is not contained in $g p\{C\}$.

Now we assume that the group $M$ (and therefore also $M_{2}$ ) is not infinite dihedral. Let $Y$ be an arbitrary element of $L_{K}=R_{K} \cap L$, where $K=F\left(\{C\} \cup\left\{W_{j}\right\}_{j \in J}\right)$. We note that if $A^{t} \in M_{2}$, where $A$ is a period and $|t| \geqslant 1$, then there exists a word $Z \in M_{2}$ such that $Z A^{t} Z^{-1} \neq A^{ \pm t}$, since otherwise it follows from [8, Lemma 34.9] that the group $M_{2}$ is either infinite cyclic or infinite dihedral.

By Lemma 3.7, a subgroup $g p\left\{C^{l}, Z\right\}$, where $Z$ is an element of $M_{2}$ such that $Z C^{l} Z^{-1} \neq C^{ \pm l}$, contains a non-dihedral period $A$ such that $F(\{C\}) \subseteq F(\{A\})$. Then by the definition of a generating mapping on $\Omega$, either $F(\{Y\}) \subseteq F(\{A\})$ or there are $W_{i_{1}}, \ldots, W_{i_{t}}, t \geqslant 1$, such that $F(\{Y\}) \subseteq F\left(\left\{A, W_{i_{1}}, \ldots, W_{i_{t}}\right\}\right)$. In the second case, we may assume that $W_{i_{s}} A W_{i_{a}}^{-1} \neq A^{ \pm 1}$ for each $s, 1 \leqslant s \leqslant t$, since otherwise by Lemma $3.4, W_{i_{s}} \in g p\{A\}$ and $F\left(\left\{W_{i_{s}}\right\}\right)=F(\{A\})$. Consider a subgroup $R_{1}=g p\left\{A, W_{i_{1}}\right\}$. By Lemma 3.7, the group $R_{1}$ contains a period $A_{1}$ such that $F\left(\left\{A_{1}\right\}\right)=F\left(\left\{A, W_{i_{1}}\right\}\right)$. Similarly, a group $R_{2}=g p\left\{A_{1}, W_{i_{2}}\right\}$ contains a period $A_{2}$ such that $F\left(\left\{A_{2}\right\}\right)=$ $F\left(\left\{A, W_{i_{1}}, W_{i_{2}}\right\}\right)$, and so on.

As a result, we obtain a period $E \in L^{\prime}=M_{2} \cap L$ such that $F(\{Y\}) \subseteq F(\{E\})$. 
Now if $|Y|>d|E|$, then by Lemma 3.7, a subgroup $g p\{E, Z\}$, where $Z$ is an element of $M_{2}$ such that $Z E Z^{-1} \neq E^{ \pm 1}$, contains a period $E_{1}$ such that $F(\{Y\}) \subseteq F\left(\left\{E_{1}\right\}\right)$ and $n|E|<\left|E_{1}\right|$. Repeating the same trick several times, we have that $L^{\prime}$ contains a period $B$ such that $F(\{Y\}) \subseteq F(\{B\})$ and $|Y|<d|B|$. Then, as in the proof of [6, Theorem A], we obtain that $Y \in L^{\prime}$ and $L^{\prime}=L_{K}$.

3) If $C \nsubseteq G_{i}$ for each $i \in I$, then by the statement of Theorem A, $f(C) \cap \Omega_{1} \neq \emptyset$. Let $a \in f(C) \cap \Omega_{1}$ and $L_{C}^{\prime}=g p\left\{c b a b^{-1} c^{-1} ; b, c \in C\right\}$. It is obvious that $L_{C}^{\prime} \leqslant L_{C}$. Now we prove that $L_{C} \leqslant L_{C}^{\prime}$. For this purpose, we may repeat the proof of assertion 6 of [6, Theorem A] if we show that the group $L_{C}^{\prime}$ is not infinite dihedral. It follows from the statement of the theorem, the definition of the relations of $G$ and [8, Lemma 34.11] that there is $b \in C$ such that $A=[a, b]$ is a free element. So it is sufficient to find a word $T \in L_{C}^{\prime}$ such that $T A T^{-1} \neq A^{ \pm 1}$.

By definition, $L_{C}$ is a normal subgroup of a subgroup $M$ and $M$ is not cyclic or infinite dihedral. Moreover, it follows from assertion 4 of the theorem that $M \leqslant R_{C}$. Hence the group $R_{C}$ is not infinite dihedral and there exists $c \in C$ such that $c \neq a$ and $c \neq b^{-1}$. Now we put $T=c b a b^{-1} c^{-1}$ and assume that $T A T^{-1}=A^{ \pm 1}$ in $G$. It follows from [8, Lemma 23.16] that this equation is also true in the group $G(1)$, and we arrive at a contradiction to the choice of $c$.

4) Let $C \in 2^{\Omega} \backslash\{\emptyset\}$ and $C \nsubseteq G_{i}$ for each $i \in I$. Then, as in 2), the group $L_{C}$ contains two distinct non-dihedral periods $A$ and $B$. By [8, Lemma 34.9], $C_{G}(A)=$ $g p\{A\}$ and $C_{G}(B)=g p\{B\}$, and by [8, Lemma 34.7], $g p\{A\} \cap g p\{B\}=\{1\}$. Hence $C_{G}\left(L_{C}\right)=\{1\}$.

It is obvious that $N_{G}\left(L_{C}\right) \supseteq R_{C}$. Let $X \in N_{G}\left(L_{C}\right)$. Then $X A X^{-1} \in L_{C}$, where $A$ is a non-dihedral period from $L_{C}$, and by [6, Lemma 3] and assertion 7 of Theorem A, $X \in R_{C}$. Thus $N_{G}\left(L_{C}\right)=R_{C}$.

5) In order to prove that a subgroup $L_{C}$ is simple if $C \nsubseteq G_{i}$ for each $i \in I$, we repeat the argument in the proof of the simplicity of the subgroup $L$ in [6, Theorem A] and consider the additional case when $M$ is a normal subgroup of $L_{C}, M$ is infinite dihedral and not contained in $G_{i}$ for each $i \in I$. Then by the proofs of [8, Theorem 35.1] and assertion 4 of Theorem A, $M$ contains a power $A^{t}$ of a period $A$ and it follows from Lemma 3.7 that $g p\left\{A^{t}\right\}$ is a normal subgroup of $R_{C}$, since otherwise $M$ contains two infinite cyclic subgroups having the trivial intersection, which contradicts the choice of $M$. Hence it follows from [8, Lemma 34.9] that the group $R_{C}$ is infinite dihedral, and we arrive at a contradiction to the fact that if $B$ and $E$ are distinct periods of some ranks such that $B, E \in R_{C}$, then by [8, Lemma 34.7], the groups $g p\{B\}$ and $g p\{E\}$ are infinite cyclic subgroups of $R_{C}$ and $g p\{B\} \cap g p\{C\}=\{1\}$.

6) Assertion 11 of Theorem A follows immediately from [8, Lemma 34.10].

7) If a subgroup $M$ of $G$ is infinite dihedral and not conjugate in $G$ to a subgroup 
of some group $G_{i}, i \in I$, then we may assume that $M$ contains a power $A^{t}$ of a period $A$ and $A^{t}$ is a product in $G$ of involutions $X$ and $Y$. By [8, Lemma 34.9], $C_{G}\left(A^{t}\right)=g p\{A\}$ and it follows from [8, Lemma 34.10] that $C_{G}(M)=\{1\}$.

Let $Z \in N_{G}(M)$. Then by [8, Lemma 34.7], $Z A^{t} Z^{-1}=A^{l}$ for some integer $l$, since every element of $M$ is either a power of $A$ or an involution. It follows from [8, Lemma 25.17] (with the correction from [7]) that $t= \pm l$, and by Lemma 3.4, we have that either $Z \in g p\{A\}$ or $Z A Z^{-1}=A^{-1}$ in $G$. In the second case, it follows from [8, Lemma 34.9] that $Z X^{-1} \in g p\{A\}$, hence $N_{G}(M)$ is an infinite dihedral group.

8) If a cyclic subgroup $M=g p\{A\}$ of $G$ is not conjugate in $G$ to a subgroup of some group $G_{i}, i \in I$, and $A$ is not a product of two involutions in $G$, then we may assume that $A$ is a power $B^{t}$ of a non-dihedral period $B$. Then by [8, Lemma 34.9 and Lemma 34.7], $N_{G}(M)=C_{G}(M)=g p\{B\}$.

It remains to prove assertion 5 of the theorem. Let $\psi$ be an automorphism of a subgroup $L_{C}$, where $C \nsubseteq G_{i}$ for each $i \in I$.

LEMma 3.8. The element $\psi(a)$ is not free in $G$ for each $a \in \Omega_{1} \cap C$.

PROOF: Assuming the contrary (and multiplying $\psi$ by an inner automorphism of $R_{C}$ ), we have that $\psi(a)=A^{t}$ for some period $A$. Then by [8, Lemma 34.7], $a$ is of infinite order in $G$. Let $b$ be an arbitrary element of $C$ such that $\{a, b\} \nsubseteq G_{i}$ for each $i \in I$, and also let $\psi(b)=T$. By raising $a$ to a suitable power, we may assume that $t>100 \zeta^{-1}$. It follows from [8, Lemma 34.10] that $b a b^{-1} \neq a^{ \pm 1}$, hence $T A T^{-1} \neq A^{ \pm 1}$. By Lemma 3.2 and [6, Lemma 3], we obtain (after multiplying $\psi$ by an inner automorphism of $R_{C}$ ) that $\psi([a, b])=S^{l}$ and $\psi(a)=B$, where $S$ is a period, $B S^{l} B^{-1} \neq S^{ \pm l}$ and $F(\{B\}) \subseteq F(\{S\})$.

There is $k \geqslant 1$ such that $k|l|>n$ and $|B|<\zeta \iota(k|l||S|)^{1 / 3}$, and by Lemma 3.5 and [6, Lemma 3], we have (after multiplying $\psi$ by an inner automorphism of $R_{C}$ ) that $\psi\left(a^{-1} A_{0} a=[a, b]^{k} a[a, b]^{2 k} a[a, b]^{3 k} a\right)=S_{1}^{r}$ and $\psi(a)=T_{1}$, where $S_{1}$ is a non-dihedral period, $T_{1} S_{1}^{r} T_{1}^{-1} \neq S_{1}^{ \pm r}$ and $F\left(\left\{T_{1}\right\}\right) \subseteq F\left(\left\{S_{1}\right\}\right)$.

Now we may choose $m$ such that $m r>0,|m|>n^{6}$ and $\left|T_{1}\right|<\iota\left(m r\left|S_{1}\right|\right)^{1 / 3}$. Then it follows from Lemma 3.6, [6, Lemma 3] and the proof of Lemma 3.1 (after multiplying $\psi$ by an inner automorphism of $\left.R_{C}\right)$ that $\psi\left(a^{-1} A_{m} a\right)=\psi\left(a\left(a^{-1} A_{0} a\right)^{m}\right)=E$ and $\psi(a)=T_{2}$, where $E$ is a non-dihedral period, $E \in L_{C},\left|T_{2}\right|<3|E|$ and $F\left(\left\{T_{2}\right\}\right) \subseteq$ $F(\{E\})$. It follows from [6, Lemma 1] and [8, Theorem 22.4] that (after multiplying $\psi$ by an inner automorphism of $R_{C}$ ) there is $T_{3} \in Y_{E}$ such that $T_{2}=T_{3} E^{p}$, where $|p| \leqslant 4$. Applying the automorphism $\psi$ to both sides of the defining relation (2.1) for $A_{m}, a$ and $k=10-p$, we obtain that

$$
T_{2}^{-1} E^{n} T_{3} E^{n+10} \ldots T_{3} E^{n+30(h-2)+10}=1,
$$

and it follows from the definition of the relations (2.1), (2.4) and (2.5) for $E$ and $T_{3}$ 
that $T_{2}$ is not a free element of $G$. But $T_{2}$ is conjugate in $G$ to a power of $A$, and this contradiction completes the proof of the lemma.

IEMMA 3.9. There exists $X \in R_{C}$ with the property that $\psi\left(G_{i} \cap L_{C}\right)=$ $X\left(G_{k(i)} \cap L_{C}\right) X^{-1}, k(i) \in I$, for each $i \in I$ and if $c \in \Omega_{1} \cap C, e \in C$ and $d \in C \cup\{1\}$ such that $\{c, e\} \nsubseteq G_{i}$ for each $i \in I$, then $\psi(c)=X c_{1} X^{-1}$ and $\psi\left(\right.$ dece $\left.^{-1} d^{-1}\right)=X d_{1} e_{1} c_{1} e_{1}^{-1} d_{1}^{-1} X^{-1}$ for some $c_{1} \in \Omega_{1} \cap C, e_{1} \in C$ and $d_{1} \in C \cup\{1\}$.

Proof: We may assume that in the statement of the lemma $d e \in \Omega_{2}$ and $d \notin$ $g p\{c\}$. It follows from assertion 4 of Theorem $\mathrm{A}$ that for each $c, d$ and $e$ from the statement of the lemma, there exist $f \in C \cup\{1\}$ and $g \in C$ such that $\{c, g\} \nsubseteq G_{i}$ for each $i \in I, f g \in \Omega_{2}$, de $\neq f g$ and $f g e^{-1} d^{-1} \neq c$ in the case $c^{2}=1$. By Lemma 3.8, we have (after multiplying $\psi$ by an inner automorphism of $R_{C}$ ) that $\psi\left([c, d e]^{n}\right)=S^{k}$ and $\psi([c, f g])=W$, where $S$ is a period, $|k|>100 \zeta^{-1}$ and $W$ is a minimal word in $G$ such that $W S^{k} W^{-1} \neq S^{ \pm k}$, since $[c, f g][c, d e]^{n}[c, f g]^{-1} \neq[c, d e]^{ \pm n}$ in $G$. Then by Lemma 3.2 and [6, Lemma 3], we obtain (after multiplying $\psi$ by an inner automorphism of $\left.R_{C}\right)$ that $\psi\left((c f g) B_{0}(c f g)^{-1}\right)=A^{l}$ and $\psi\left([c, d e]^{n}\right)=B$, where $A$ is a non-dihedral period, $B A^{l} B^{-1} \neq A^{ \pm l}$ and $F(\{B\}) \subseteq F(\{A\})$, since $B_{0}$ is a non-dihedral period of $G$.

We choose an integer $m$ such that $m l>0,|m|>n^{6}$ and $|B|<\iota(m l|A|)^{1 / 3}$. Then it follows from Lemma 3.6, [6, Lemma 3] and the proof of Lemma 3.1 (after multiplying $\psi$ by an inner automorphism of $\left.R_{C}\right)$ that either $\psi\left([c, d e]^{n}\right)=[u, v y]^{n}$ in $G$ for some $u \in \Omega_{1} \cap C, y \in C$ and $v \in C \cup\{1\}$ such that $\{u, y\} \nsubseteq G_{s}$ for each $s \in I$ and $v y \in \Omega_{2}$, or $\psi\left((c f g) B_{m}(c f g)^{-1}\right)=E$ and $\psi\left([c, d e]^{n}\right)=T$, where $E$ is a non-dihedral period, $E \in L_{C},|T|<3|E|$ and $F(\{T\}) \subseteq F(\{E\})$. It follows from [6, Lemma 1] and [8, Theorem 22.4] that (after multiplying $\psi$ by an inner automorphism of $R_{C}$ ) there is $T_{1} \in Y_{E}$ such that $T=T_{1} E^{p}$, where $|p| \leqslant 4$. Applying the automorphism $\psi$ to both sides of the defining relation (2.2) (conjugated by the element $c f g$ ) for $(c f g) B_{m}(c f g)^{-1},[c, d e]^{n}$ and $k=10-p$, we obtain that

$$
T^{-1} E^{n} T_{1} E^{n+20} \ldots T_{1} E^{n+30(h-2)+20}=1,
$$

and it follows from the definition of the relations (2.2), (2.6) and (2.7) for $E$ and $T_{1}$ that again $\psi\left([c, d e]^{n}\right)=[u, v y]^{n}$ for some $u \in \Omega_{1} \cap C, y \in C$ and $v \in C \cup\{1\}$ such that $\{u, y\} \nsubseteq G$, for each $s \in I$ and $v y \in \Omega_{2}$.

By Lemma $3.8[8$, Lemma 34.7], we have that $\psi([c, d e])=[u, v y]$. It follows from Lemma 3.8 that (after multiplying $\psi$ by an inner automorphism of $\left.R_{C}\right) \psi(c)=c_{1}$ and $\psi\left(\right.$ dece $\left.{ }^{-1} d^{-1}\right)=U c_{1} U^{-1}$ for some $c_{1} \in \Omega_{1} \cap C$ and $U \in R_{C}$. Then there is a reduced circular diagram $\Delta$ (of some rank) for the conjugacy of $\left[c_{1}, U\right]$ to $[u, v y]$. Pasting together the subpaths with labels $U$ and $U^{-1}$, we arrive at a diagram $\Delta^{\prime}$ on a sphere with three holes with contour labels equal to $c_{1}, c_{1}^{-1}$ and $[u, v y]^{-1}$. The removal of 
$j$-pairs from $\Delta^{\prime}$ gives a reduced diagram $\Delta_{0}$. By [8, Theorem 22.1], $r\left(\Delta_{0}\right)=0$. Hence we obtain that $c_{1}=u^{\delta}$ and $U=Z_{1}(v y)^{\delta} Z_{2}$, where $|\delta|=1, Z_{i} \in G$ and $\left[c_{1}, Z_{i}\right]=1$ for $i=1,2$. Therefore, we have that $\psi(c)=c_{1}$ and $\psi\left(\right.$ dece $\left.^{-1} d^{-1}\right)=Z d_{1} e_{1} c_{1} e_{1}^{-1} d_{1}^{-1} Z^{-1}$ for some $e_{1} \in C$ and $d_{1} \in C \cup\{1\}$ such that $d_{1} e_{1} \in \Omega_{2}$, where $Z \in G,\left[c_{1}, Z\right]=1$ and $Z=Z_{d, e}$ depends, in general, on the choice of $e$ and $d$. Moreover, by [8, Lemma 34.10], $\left\{c_{1}, Z_{d, e}\right\} \subseteq G$, for some $s \in I$.

Suppose that $Z_{d, e} \neq Z_{f, g}$ for some $f \in C \cup\{1\}$ and $g \in C$ such that $f g \in \Omega_{2}$ and $f \notin g p\{c\}$. Then we may assume that $f g e^{-1} d^{-1} \notin g p\{c\}$, and by the previous considerations, $\psi\left([c, d e]^{n}\left[[c, d e]^{n},[c, f g]\right]^{m}\right),|m|>n^{6}$, is conjugate in $G$ to $\left[c_{1}, d_{1} e_{1}\right]^{n}\left[\left[c_{1}, d_{1} e_{1}\right]^{n},\left[c_{1}, k l\right]\right]^{m_{1}}$, where $\left|m_{1}\right|>n^{6}, k \in C \cup\{1\}, l \in C,\left\{c_{1}, l\right\} \nsubseteq G_{s}$ for each $s \in I$ and $k l \in \Omega_{2}$. It follows from the proof of Lemma 3.1 and the choice of defining relations in $G$ that $\left[Z_{d, e}\left[c_{1}, d_{1} e_{1}\right]^{n} Z_{d, e}^{-1}, Z_{f, g}\left[c_{1}, f_{1} g_{1}\right] Z_{f, g}^{-1}\right]$ is conjugate to $\left[\left[c_{1}, d_{1} e_{1}\right]^{n},\left[c_{1}, k l\right]\right]$ in the group $G(1)$, which is impossible in our case. Thus we have that $Z_{d, e}=Z_{c}$ for each $d \in C \cup\{1\}$ and $e \in C$ such that $d e \in \Omega_{2}$, where $\left\{Z_{c}, c_{1}\right\} \subseteq G_{s}$ for some $s \in I$ and $\left[c_{1}, Z_{c}\right]=1$.

We note that if $a \in G_{i} \cap L_{C}$ and $\psi(a) \in G_{k(i)} \cap L_{C}$ for some $i, k(i) \in I$, then $\psi\left(G_{i} \cap L_{C}\right)=G_{k(i)} \cap L_{C}$, since by Lemma 3.8 and the proof of [8, Theorem 35.1] if $b \in G_{i} \cap L_{C}$ and $\psi(b) \notin G_{k(i)}$, then $\psi(a b)$ is not conjugate to an element of $\Omega$, contradicting Lemma 3.8.

We may assume (after multiplying $\psi$ by an inner automorphism of $R_{C}$ ) that $\psi(c)=c_{1}$ and $Z_{c}=1$. It remains to prove that $\psi(e) \in \Omega_{1} \cap C$ and $Z_{e}=1$ for each $e \in \Omega_{1} \cap C$. Let $e \in \Omega_{1} \cap C$ such that $\{c, e\} \subseteq G_{s}$ for some $s \in I$ and $e \neq c$, and also let $d$ be an arbitrary element of $C$ with $\{e, d\} \nsubseteq G_{i}$ for each $i \in I$. By the previous considerations, $\psi(e)=e_{1} \in \Omega_{1} \cap C$. Then

$$
\psi\left(d c e d^{-1}\right)=\psi\left(d c d^{-1}\right) \psi\left(d e d^{-1}\right)=d_{1} c_{1} d_{1}^{-1} Z_{e} d_{2} e_{1} d_{2}^{-1} Z_{e}^{-1}
$$

for some $d_{1}, d_{2} \in C$, hence $Z_{e}=1$, since otherwise $\psi\left(d c e d^{-1}\right)$ is a free element of $G$ and we arrive at a contradiction to Lemma 3.8.

Now we consider the case when $e$ is an arbitrary element of $\Omega_{1} \cap C$ such that $\{c, e\} \nsubseteq G_{i}$ for each $i \in I$ (if such an $e$ exists). Repeating the considerations from the beginning of the proof of the lemma for an element $[c, e]$, we obtain that $\psi(e)=e_{1} Z$ for some $e_{1} \in \Omega_{1} \cap C$ and $Z \in G$ such that $\left[c_{1}, Z\right]=1$. By [8, Lemma 34.10], $\left\{Z, c_{1}\right\} \subseteq G_{s}$ for some $s \in I$, hence $Z=1$, since otherwise $\psi(e)$ is a free element of $G$, which contradicts Lemma 3.8. Similary, we have that $\psi(c)=Z_{e} c_{2} Z=c_{1}$, where $\left\{Z_{e}, Z, e_{1}\right\} \subseteq G_{j}$ for some $j \in I$ and $c_{2} \in C$, which is possible only if $Z_{e}=Z=1$ and $c_{1}=c_{2}$.

The proof of the lemma is complete.

Lemma 3.10. There exists $X \in R_{C}$ such that $\psi(a)=X a X^{-1}$ for each $a \in$ 
$\Omega_{1} \cap C$.

Proof: By Lemma 3.9, we have (after multiplying $\psi$ by an inner automorphism of $R_{C}$ ) that $\psi\left(G_{i} \cap L_{C}\right)=G_{k(i)} \cap L_{C}, k(i) \in I$, for each $i \in I$. Let $s$ be the minimal element of $I$ such that $G_{i} \cap L_{C} \neq\{1\}$ and $s \neq k(s)$. Hence $s<k(s)$ and there exists $p \in I$ such that $k(p)=s$ with $p>s$. Let $c$ and $e$ be arbitrary nontrivial elements of $G_{s} \cap L_{C}$ and $G_{p} \cap L_{C}$, respectively. By assertion 4 of Theorem A, the group $L_{C}$ is not dihedral and there are $f \in C \cup\{1\}$ and $g \in C$ such that $\{c, g\} \nsubseteq G_{j}$ for each $j \in I, f g \in \Omega_{2}, e \neq f g$ and $f g e^{-1} \neq c$ in the case $c^{2}=1$. Let $\psi(c)=c_{1}, \psi(e)=e_{1}$ and $\psi\left(f g c g^{-1} f^{-1}\right)=f_{1} g_{1} c_{1} g_{1}^{-1} f_{1}^{-1}$. Then $c<e$ and $c_{1}>e_{1}$, and applying the automorphism $\psi$ to both sides of the defining relation (2.3) (conjugated by the element $c f g$ ) for $(c f g) B_{m}(c f g)^{-1}=[c, e]^{n}\left[[c, e]^{n},[c, f g]\right]^{m}$ and $T=[c, e]$, where $m>n^{6}$, we obtain that

$$
c_{1}\left(B_{m}^{\prime}\right)^{n}\left[c_{1}, e_{1}\right]\left(B_{m}^{\prime}\right)^{n+25} \ldots\left[c_{1}, e_{1}\right]\left(B_{m}^{\prime}\right)^{n+30(h-2)+25}=1,
$$

where $B_{m}^{\prime}=\psi\left((c f g) B_{m}(c f g)^{-1}\right)$, and it follows from the definition of the relation (2.3) for $\left(c_{1} f_{1} g_{1}\right)^{-1} B_{m}^{\prime}\left(c_{1} f_{1} g_{1}\right)$ and $T=\left(c_{1} f_{1} g_{1}\right)^{-1}\left[c_{1}, e_{1}\right]\left(c_{1} f_{1} g_{1}\right)$ that $c_{1}=e_{1} c_{1} e_{1}^{-1}$, which contradicts $\left[8\right.$, Lemma 34.10]. Thus $\psi\left(G_{i} \cap L_{C}\right)=G_{i} \cap L_{C}$ for each $i \in I$.

Let $c$ be the minimal element of $\Omega_{1} \cap C$ such that $\psi(c) \neq c$. Hence $c<\psi(c)=$ $c_{1}, c_{1} \in \Omega_{1} \cap C$, and there exists $d \in \Omega_{1} \cap C$ such that $\psi(d)=c$ and $c<d$. By assertion 4 of Theorem A, there is $e \in C$ such that $\{c, e\} \nsubseteq G_{i}$ for each $i \in I$. Applying the automorphism $\psi$ to both sides of the defining relation (2.4) (conjugated by the element ce) for $(c e) B_{m}(c e)^{-1}=[c, d e]^{n}\left[[c, d e]^{n},[c, e]\right]^{m}$ and $T=c$, where $m>n^{6}$, we have that

$$
\left(c_{1} e_{1}\right) c_{1}\left(c_{1} e_{1}\right)^{-1}\left(B_{m}^{\prime}\right)^{n} c_{1}\left(B_{m}^{\prime}\right)^{n+10} \ldots c_{1}\left(B_{m}^{\prime}\right)^{n+30(h-2)+10}=1
$$

where $e_{1} c_{1} e_{1}^{-1}=\psi\left(e c e^{-1}\right)$ and $B_{m}^{\prime}=\psi\left((c e) B_{m}(c e)^{-1}\right)$. It follows from the definition of the relation (2.4) for $\left(c_{1} e_{1}\right)^{-1} B_{m}^{\prime}\left(c_{1} e_{1}\right)$ and $T=\left(c_{1} e_{1}\right)^{-1} c_{1}\left(c_{1} e_{1}\right)$ that $c=c_{1}$, and this contradiction completes the proof of the lemma.

Lemma 3.11. There exists $X \in R_{C}$ such that $\psi\left(\right.$ dece $\left.{ }^{-1} d^{-1}\right)=X$ dece $^{-1} d^{-1} X^{-1}$ for each $c \in \Omega_{1} \cap C, e \in C$ with $\{c, e\} \nsubseteq G_{i}$ for each $i \in I$ and $d \in C \cup\{1\}$.

Proof: By Lemmas 3.9 and 3.10, we have (after multiplying $\psi$ by an inner automorphism of $R_{C}$ ) that $\psi\left(d e c e^{-1} d^{-1}\right)=d_{1} e_{1} c e_{1}^{-1} d_{1}^{-1}$, where $e_{1} \in C,\left\{e_{1}, c\right\} \nsubseteq G_{i}$ for each $i \in I$ and $d_{1} \in C \cup\{1\}$. We may assume that $d e, d_{1} e_{1} \in \Omega_{2}$.

Let $d e$ be the minimal element of $\Omega_{2}$ such that $d e c e^{-1} d^{-1} \in L_{C}$ and $\psi\left(\right.$ dece $\left.e^{-1} d^{-1}\right)$ $=d_{1} e_{1} c e_{1}^{-1} d_{1}^{-1} \neq d e c e^{-1} d^{-1}$. Hence $d e<d_{1} e_{1}$ and there exists $f g \in \Omega_{2}$ such that $f g c g^{-1} f^{-1} \in L_{C}$, de $<f g$ and $\psi\left(f g c g^{-1} f^{-1}\right)=d e c e^{-1} d^{-1}$. We have that $f g e^{-1} d^{-1} \neq c$, since otherwise

$$
d e c e^{-1} d^{-1}=\psi\left(f g c g^{-1} f^{-1}\right)=c \psi\left(d e c e^{-1} d^{-1}\right) c^{-1}=c d_{1} e_{1} c e_{1}^{-1} d_{1}^{-1} c^{-1}
$$


and by $\left[8\right.$, Lemma 34.10], $\left\{e^{-1} d^{-1} c d_{1} e_{1}, c\right\} \subseteq G$, for some $s \in I$, which is impossible.

By applying the automorphism $\psi$ to both sides of the defining relation (2.3) (conjugated by the element $c f g$ ) for $T=[c, d e]^{2}$ and

$$
(c f g) B_{m}(c f g)^{-1}=[c, d e]^{n}\left[[c, d e]^{n},[c, f g]\right]^{m},
$$

where $m>n^{6}$, we obtain that

$$
d_{1} e_{1} c e_{1}^{-1} d_{1}^{-1}\left(B_{m}^{\prime}\right)^{n}\left[c, d_{1} e_{1}\right]^{2}\left(B_{m}^{\prime}\right)^{n+25} \ldots\left[c, d_{1} e_{1}\right]^{2}\left(B_{m}^{\prime}\right)^{n+30(h-2)+25}=1,
$$

where $B_{m}^{\prime}=\psi\left((c f g) B_{m}(c f g)^{-1}\right)$, and it follows from the definition of the relation (2.3) for $(c d e)^{-1} B_{m}^{\prime}(c d e)$ and $T=(c d e)^{-1}\left[c, d_{1} e_{1}\right]^{2}(c d e)$ that $d_{1} e_{1} c e_{1}^{-1} d_{1}^{-1}=d e c e^{-1} d^{-1}$, which contradicts our assumption.

The proof of the lemma is complete.

Now assertion 5 of Theorem A about Aut $L_{C}$ follows from Lemma 3.11 and assertion 6 of Theorem A. The assertion about regular automorphisms of $L_{C}$ follows immediately from [8, Lemma 34.10].

The proof of Theorem $A$ is complete.

\section{REFERENCES}

[1] G. Higman, B.H. Neumann and H. Neumann, 'Embedding theorems for groups', J. London Math. Soc. 24 (1949), 247-254.

[2] B.H. Neumann, 'Some remarks on infinite groups', J. London Math. Soc. 12 (1937), 120-127.

[3] V.N. Obraztsov, 'An embedding theorem for groups and its applications', Mat. Sb. 180 (1989), 529-541.

[4] V.N. Obraztsov, 'Embedding schemes for groups and some applications', (deposited VINITI 8 February 1990, no. 724-B90).

[5] V.N. Obraztsov, 'On infinite complete groups', Comm. Algebra 22 (1994), 5875-5887.

[6] V.N. Obraztsov, 'A new embedding scheme for groups and some applications', J. Austral. Math. Soc. (to appear).

[7] A.Yu. Ol'shanskii, 'On thrifty embeddings of groups', Vestnik Moskovsk. Univ. 2 (1989), 28-34.

[8] A.Yu. Ol'shanskii, Geometry of defining relations in groups, (in Russian) (Nauka, Moscow, 1989); (English translation: North Holland, 1991).

[9] S. Shelah, 'On a problem of Kurosh, Jonsson groups, and applications', in Word Problems II: the Oxford book, (S. Adian, W.W. Boone and G. Higman, Editors) (North Holland, 1980), pp. 373-394.

\footnotetext{
Department of Mathematics

The University of Melbourne

Parkville, Vic 3052

Australia
} 